\title{
Effect of Extenders with TCG and DMSO on the Viability of Rabbit Sperm
}

\author{
Yeol Eo 2,\#, Sang Hwan Kim", Seong-Gyu Bang ${ }^{3}$, Min-Gee Oh ${ }^{3}$, Chan-Hee Park ${ }^{3}$ and \\ Jong Taek Yoon ${ }^{1,3, *}$ \\ ${ }^{1}$ Institute of Genetic Engineering, Hankyong National University, Anseong 17579, Korea \\ ${ }^{2}$ Major in the Animal Biotechnology, Graduate School of Future Convergence Technology, Hankyong National \\ University, Anseong 17579, Korea \\ ${ }^{3}$ Department of Animal Life Science, Hankyong National University, Anseong 17579, Korea
}

Received May 31, 2019

Revised June 20, 2019

Accepted June 27, 2019

\section{*Correspondence \\ Jong Taek Yoon \\ Department of Animal Life Science, Hankyong \\ National University, 327 Jungang-ro, Anseong \\ 17579, Korea \\ Tel: +82-31-670-5094 \\ Fax: +82-31-675-8265 \\ E-mail: jtyoon@hknu.ac.kr \\ ORCID \\ https://orcid.org/0000-0002-9408-8239}

"The authors contributed equally to this work.

\begin{abstract}
The purpose of this study was to evaluate the effect of addition of ethylene glycol, glycerol and sucrose to TCG (Tris, Citric Acid, Glucose, Egg Yolk) and DMSO Frozen. The extender containing Egg yolk concentration (10\%, 20\%) affects viability and acrosome morphology of rabbit sperm. Sperm viability was then assessed for the freezing extenders TCGD (Tris + Citricacid + Glucose + DMSO), TCGED (Tris + Citricacid + Glucose + Egg yolk + DMSO), TCGGD (Tris + Citricacid + Glucose + Glycerol + DMSO) and TCGSD Tris + Citricacid + Glucose + Sucrose + DMSO) during thawing at $38^{\circ} \mathrm{C}$. for 20 seconds, respectively. TCG $+10 \%$ egg yolk (viability: $77.0 \pm 0.8$, NAl: $73.3 \pm$ 0.9 ) was significantly (sperm viability and normal acrosome interaction (NAI)) higher than TCG $+20 \%$ egg yolk $(70.7 \pm 1.1,70.0 \pm 0.9)$ in the sperm normalcy analysis according to the yolk concentration. TCGGD (53.4 $\pm 0.1,62.3 \pm 0.4)$, TCGSD (61.3 $\pm 0.0,67.1 \pm 0.1$ ) sperm viability and normal acrosome interaction (NAl) in frozen spermatozoa are TCGD $(46.4 \pm 2.8$ and $56.3 \pm 1.4)$ and TCGED $(23.0 \pm 1.1$ and 54.6 \pm 1.4 ) extenders was thawed at $38^{\circ} \mathrm{C}$ for 20 seconds. According to the results from each frozen bulking agent, sperm membrane integrity by hypotonic swelling test (HOST) analysis in TCGGD (59.8 \pm 0.7$)$, TCGSD $(59.3 \pm 0.5)$ was significantly high compared to other experimental groups $(p<0.05)$. In conclusion, these results suggested that TCGGD and TCGSD extenders enhance survivability of rabbit sperm after frozenthawing.
\end{abstract}

Keywords: extender, HOST, rabbit, sperm, TCG

\section{INTRODUCTION}

The market for rabbit meat industry is known to be an important source for future food resources. In particular, United Nations Food and Agriculture Organization (FAO) focuses on rabbit meat as a solution to the world food problem (Foote and Carney, 2000). However, studies on the improvement and mass production of rabbits had not been completed, and research on the application of frozen semen had also been lacking. The sperm of rabbits was suggested to the differential morphological and physiological on the sperms of the other economic animals. The extender causes cold shock on sperm acrosome or survival rate during frozen (Watson, 2000). Semen freezing with egg yolk and Tris, Citric acid, Glucose (TCG) was known as a commonly used extenders for freezing preser- 
vation of rabbit sperm. There were many studies on freezing method by adding glycerol, dimethylsulfoxide (DMSO) and sucrose to the second extender with freezing extender. However, studies on the effect of buffer concentration and composition on sperm viability are insufficient (Cortell and Viudes, 2008). In particular, the frozen of sperm using glycerol is highly effective in preventing frostbite from frozen in many animal sperm (Polge et al., 1949). Especially, rabbit sperm have been difficult to freeze successfully (Hanada and Nagase, 1980; Chen et al., 1989a, 1989b; Bamba, 1990; Chen and Foote, 1994). The incorporation of detergents in seminal extenders has improved the quality of cryopreserved sperm from boars (Pursel et al., 1978), rams (El-Alamy and Foote, 2000), bulls (Arriola and Foote, 1987), dogs (Rota et al., 1997), and mice (Dewit et al., 2000). No similar studies have been reported for rabbit sperm. However, the concentration of glycerol in some animals (bovine and pigs etc.) is controlled in the toxicity about sperm. In animal species including chickens, sheep, pigs and horse's glycerol had been reported to reduce fertilization rate and capacitation (Swanda et al., 1964; Hammerstedt et al., 1992). Therefore, this study analyzed the viability of spermatozoa according to the freezing extender of rabbit sperm.

\section{MATERIALS AND METHODS}

\section{Semen collection}

The rabbit semen collection was performed by using a self-made sperm sampler at intervals of one week. The semen sample was quantitated using a micropipette, and $10 \mu \mathrm{L}$ of diluted semen was collected from the sampler into a chamber and then diluted with a primary agent to obtain a sperm concentration of $4 \times 10^{5} \mathrm{sperm} / \mathrm{mL}$. The

Table 1. Composition of first extenders for semen dilution

\begin{tabular}{lcc}
\hline \multirow{2}{*}{ First extender } & Composition in extender $100 \mathrm{~mL}(\mathrm{~g})$ \\
\cline { 2 - 3 } & TCG10 & TCG20 \\
\hline Tris & 3.028 & 3.028 \\
Citric acid & 1.690 & 1.690 \\
Glucose & 0.847 & 0.847 \\
OSM & 303 & 303 \\
pH & 6.9 & 6.9 \\
Egg yolk & $10 \mathrm{~mL}$ & $20 \mathrm{~mL}$ \\
\hline
\end{tabular}

*TCG10 (Tris, Citric, Glucose and 10\% egg yolk), TCG20 (Tris, Citric, Glucose and 20\% egg yolk), OSM (osmoles mater). extender was stored in a thermostat warmed at $37^{\circ} \mathrm{C}$ and was either used for artificial insemination within 2 hours or transferred to a laboratory for freezing.

\section{Preparation of extender}

The first extender prepared with Tris $250 \mathrm{mM}$ (T-6791, Sigma, USA), Citric acid 88 mM (C-2404, Sigma, USA), and Glucose $47 \mathrm{mM}$ (G-7021, Sigma, USA).

The osmotic pressure of the first extender was adjusted to Osmol and the $\mathrm{pH}$ was adjusted to 7.0 . Ten and $20 \%$ of the egg yolk was added to the first extender and it was disclosed to the experiment comparing the viability of the sperm with the long term preservation (Table 1).

The second extender composed of $10 \%$ of egg yolk, 3.5 M of DMSO (D-2650, SIGMA, USA), 3\% of ethylene glycol, $3.5 \mathrm{M}$ of DMSO, 3\% of glycerol and 3.5 M, DMSO, $0.1 \mathrm{M}$ sucrose and 3.5 M DMSO in primary extender (Table 2).

\section{Low-temperature storage and examination of survival rate of sperm}

One of treatment groups was in a double boiler at 16 to $37^{\circ} \mathrm{C}$ and another of those was from 4 to $37^{\circ} \mathrm{C}$. Semen dilution cooled down at $4^{\circ} \mathrm{C}$ and $16^{\circ} \mathrm{C}$ for 2 hour and kept five days. Survival rate was measured at 12 hour intervals at $37^{\circ} \mathrm{C}$ with a $100 \times$ magnification microscope.

\section{Semen freezing and examination of survival rate of sperm}

The semen transferred to the laboratory was dispensed by experimental treatments. The hot water at $37^{\circ} \mathrm{C}$ was placed in a beaker and the semen injected into the beaker was stored in a refrigerator at $4^{\circ} \mathrm{C}$ for 2 hour. The equilibrated semen was preliminarily frozen in $0.5 \mathrm{~mL}$ straw from $10 \mathrm{~cm}$ above the surface of liquid nitrogen, frozen by immersion in liquid nitrogen stored until the survival rate

Table 2. Composition of second extenders for semen dilution

\begin{tabular}{lcccc}
\hline \multirow{2}{*}{ Second extender } & \multicolumn{4}{c}{ Composition in extender $(\mathrm{g} / \mathrm{mL})$} \\
\cline { 2 - 5 } & TCGD & TCGED & TCGGD & TCGSD \\
\hline Ethylene glycerol & & 1.5 & & \\
DMSO & 13.7 & 13.7 & 13.7 & 13.7 \\
Glycerol & & & 1.5 & \\
Sucrose & & & & 1.7 \\
\hline
\end{tabular}

*TCGD (Tris, Citric, Glucose and DMSO), TCGED (Tris, Citric, Glucose, Ethylene glycerol and DMSO), TCGGD (Tris, Citric, Glucose, Glycerol and DMSO), TCGGD (Tris, Citric, Glucose, Sucrose and DMSO). 
was checked. The frozen semen was thawed at $38^{\circ} \mathrm{C}$ for 20 seconds.

\section{Survival rate and acrosomal normaliztion}

De Leeuw (1991) was improved and frozen semen was melted at $38^{\circ} \mathrm{C}$ for 20 seconds. Then, $100 \mu \mathrm{L}$ of sperm dilution and $600 \mu \mathrm{L}$ of $0.3 \%$ BSA were mixed and centrifuged at $1500 \mathrm{rpm}$ for 5 minutes. After centrifugation, the supernatant was removed, Hoechst 33258 was added well at a concentration of $100 \mu \mathrm{g} / \mathrm{mL}$ and stored in the dark for 1 minute 30 seconds, then mixed with $10 \mu \mathrm{L}$ of $4 \%$ paraformaldehyde. On the slides, $50 \mu \mathrm{L}$ of the treated semen was covered with cover grass, and then 200 of sperm were observed at $\times 400$ magnification. Two hundred sperm were counted and dyed sperm were counted as dead sperm. For analysis of acrosomal integrity of sperm, semen was stained with Coomassie Brilliant Blue (R-266, TNT, Republic of Korea) as described previously (Larson and Miller, 1999). Frozen-semen thawed at $38^{\circ} \mathrm{C}$ for 20 seconds, and then $100 \mu \mathrm{L}$ of frozen-thawed semen was centrifuged at $1500 \mathrm{rpm}$ for 5 minutes. The supernatant was removed. $50 \mu \mathrm{L}$ of $1 \times$ PBS was added to $10 \mu \mathrm{L}$ of sperm pellet and centrifugation was performed twice at $1500 \mathrm{rpm}$ for 5 minutes.

The semen was diluted in $500 \mu \mathrm{L}$ of $1 \times$ PBS and $50 \mu \mathrm{L}$ of the solution was placed on a slide and dried at $37^{\circ} \mathrm{C}$. The cells were stained by $0.25 \%$ Coomassie Briliant Blue (R-266) solution for 5 minutes and washed twice with $1 \times$ PBS. Acrosomal normality was determined by observing the presence or absence of blue dyeing and the degree of staining of the sperm's head in a microscope at $\times 400$ magnification. 300 sperm were counted and converted into percentages. Sperm dyed in blue were classified as normal sperm.

\section{Plasma membrane function test}

Plasma membrane function test of sperm was performed as described previously (Jeyendran RS, 1984). The frozen semen thawed in water at $38^{\circ} \mathrm{C}$ for 20 seconds and then $100 \mu \mathrm{L}$ of frozen-thawed semen was centrifuged at $1500 \mathrm{rpm}$ for 5 minutes. After removal of the supernatant, added $1 \mathrm{~mL}$ of hypoosmotic solution and stored at $37^{\circ} \mathrm{C}$ for 30 minutes. $50 \mu \mathrm{L}$ of treated semen dilution was placed on a slide and more than number of 300 sperm were counted to analyzed the degree of fin and buoyancy of the tail with $\times 400$ magnification.

\section{Statistical analysis}

Percentage of motility, viability and survivability of spermatozoa of among treatment groups were compared by Duncan's multiple range test in application of ANOVA and GLM using SAS package (version 9.1).

\section{RESULTS}

\section{Low temperature preservation of rabbit semen}

The collected rabbit semen was stored at $16^{\circ} \mathrm{C}$ and $4^{\circ} \mathrm{C}$ for 72 hours according to the low temperature preserva-

Table 3. Addition effect of Tris in freezing extender on frozen-thawed sperm motility and viability of rabbit spermatozoa (Mean \pm SD)

\begin{tabular}{|c|c|c|c|c|c|c|c|c|}
\hline \multirow{2}{*}{$\begin{array}{l}\text { Type of semen } \\
\text { extenders }\end{array}$} & \multirow{2}{*}{ Temperature $\left({ }^{\circ} \mathrm{C}\right)$} & \multicolumn{7}{|c|}{ Preservation periods $(\mathrm{h})$} \\
\hline & & 0 & 12 & 24 & 36 & 48 & 60 & 72 \\
\hline TCG & 4 & $90 \%$ & $78.0 \pm 0.7$ & $51.1 \pm 1.6$ & $23.4 \pm 0.6$ & $16.5 \pm 0.4$ & 0.0 & 0.0 \\
\hline \multirow[t]{2}{*}{ TCGE } & 4 & $90 \%$ & $78.5 \pm 0.4$ & $72.7 \pm 0.6$ & $64.4 \pm 1.5$ & $53.5 \pm 1.2$ & $26.8 \pm 1.5$ & $17.8 \pm 1.5$ \\
\hline & 16 & $90 \%$ & $85.8 \pm 1.2$ & $81.7 \pm 1.1$ & $76.1 \pm 1.2$ & $60.8 \pm 1.2$ & $39.2 \pm 0.1$ & $19.3 \pm 1$ \\
\hline \multirow[t]{2}{*}{ TCGEC } & 4 & $90 \%$ & $78.75 \pm 1.8$ & $70 \pm 1.8$ & $60 \pm 0.3$ & $53.7 \pm 3.5$ & $30.5 \pm 1.8$ & $10.5 \pm 0.3$ \\
\hline & 16 & $90 \%$ & $85 \pm 1.5$ & $78.75 \pm 1.8$ & $66.2 \pm 1.8$ & $60 \pm 0.3$ & $30 \pm 1.5$ & $10 \pm 0.3$ \\
\hline \multirow[t]{2}{*}{ TCGM } & 4 & $90 \%$ & $78.0 \pm 0.7$ & $51.1 \pm 1.6$ & $23.4 \pm 0.6$ & $16.5 \pm 0.4$ & 0.0 & 0.0 \\
\hline & 16 & $95 \%$ & $78.3 \pm 0.8$ & $55.6 \pm 0.9$ & $23.0 \pm 0.1$ & $19.5 \pm 1.6$ & $16.5 \pm 0.2$ & 0.0 \\
\hline TCGPEM & 4 & $85 \%$ & $62.3 \pm 1.1$ & $63.5 \pm 1.6$ & $51.2 \pm 1.8$ & $41 \pm 1.4$ & $32.7 \pm 1.0$ & 0.0 \\
\hline
\end{tabular}

*TCG (Tris, Citric and Glucose), TCGE (Tris, Citric, Glucose and Ethylene glycerol), TCGP (TCG + PVP), TCGec (TCG + Ethyleneclycol), TCGM (TCG +Mercaptoethanol), TCGPEM (TCG + PVP + Egg yolk+Mer captoethanol). 
tion extender prepared by the research team. The survival rate and the change of vitality were measured.

Table 3 shown the survival rates of low-temperature preserved agent by TCG (Tris + Citricacid + Glucose) dilution, TCGE (TCG + Egg yolk), TCGP (TCG + PVP), TCGec (TCG + Ethyleneclycol), TCGM (TCG + Mercaptoethanol), and TCGPEM (TCG + PVP + Eggyolk + Mercaptoethanol).

As a result of evaluating the cold storage property according to each frozen agent by temperature, the survival rate was higher at $16^{\circ} \mathrm{C}$ than at $4^{\circ} \mathrm{C}$ in all the low temperature frozen agent. In the extenders analysis, survival rate of the samples was $0.0 \%$ at 72 hours on $16^{\circ} \mathrm{C}$. The survival rate was $14.6 \% \pm 1.6 \%$ in TCGP, $10 \pm 0.3 \%$ in TCGec, $0.0 \%$ in TCGM, $10 \pm 1.4 \%$ in TCGPEM, and the survival rate of TCGM on $19.3 \pm 1 \%$ was higher than other frozen agents (Table 3).

\section{Survival and vitality of rabbit sperm according to the egg yolk addition concentration}

In order to investigate the effect of egg yolk addition on the vitality of sperm during low - temperature storage of rabbit sperm, the addition of egg yolk was added to a concentration of $10 \%, 20 \%$ according to result preserving in TCGE cold storage agent at $16^{\circ} \mathrm{C}$. The results are shown in Table 4 . From 12 to 84 hours, survival rate of TCGE10 with $10 \%$ egg yolk was $85.8 \pm 1.1 \%$ at 12 hours, $77 \pm 0.8 \%$ at 48 hours, $67.5 \pm 1.9 \%$ at 36 hours, and 48.2 $\pm 1.2 \%$ at 60 hours and $36.0 \pm 0.9 \%$ at 72 hours. The survival rate of TCGE20 was $80.8 \pm 1.2 \%$ at 12 hours, $76.7 \pm 1.1 \%$ at 24 hours, $70.1 \pm 1.2 \%$ at 30 hours, 60.8 $\pm 1.2 \%$ at 48 hours and $39.2 \pm 0.1 \%$ at 60 hours and 19.3 $\pm 1 \%$ after 72 hours. the low temperature frozen agent of TCGE10 was significantly $(p<0.05)$ higher than the low temperature frozen agent of TCGE20. In addition, in the plasma membrane test of Table 5, TCGE10 showed $73 \pm$ $1.4 \%$ of plasma membrane function normal. TCGE20 was $70 \pm 1.4 \%$ of plasma membrane function normal and the low temperature frozen agent of TCGE10 was significantly higher than TCGE20 $(p<0.05)$.

\section{Ability rate of sperm according to frozen extender}

To analyze the effect of extender on viability and acrosome test after frozen thawing, added 3.5 M of DMSO, 3\% ethylene glycol, 3.5 M DMSO in dilution (below TCGED), $3 \%$ glycerol, supplement 3.5 M DMSO (below TCGGD), $0.1 \mathrm{M}$ sucrose, 3.5 M DMSO (below TCGSD) were used for the experiment in the semen of rabbits diluted with cold extenders. The survivability and acrosome stability of the rabbit semen after freezing and thawing according to each frozen extenders were shown in Fig. 1 and Table 6. The results of acrosome normality and the survival rate of rabbit sperm and was analyzed by Hoechst fluorescence staining in classifying fluorescently stained sperm is dead sperm and unstained sperm is living sperm. High survival rates were identified in TCGGD and TCGSD semen frozen agent. Based on the results of fluorescence staining analysis, the survival and acrosome stability of rabbit sperm in frozen agent were evaluated using computer assisted sperm analysis system. In TCGGD, $53.4 \pm 0.1 \%$ and $62.3 \pm$ $0.4 \%, 53.4 \pm 0.1 \%$ and $62.3 \pm 0.4 \%$ in TCGGD, $61.3 \pm$ $0 \%$ and $67.1 \pm 0.1 \%$ in TCGSD. In TCGD $46.4 \pm 2.8 \%$ and $56.3 \pm 1.4 \%$ was significantly higher than $23.0 \pm 1.1 \%$ and $54.6 \pm 1.4 \%$ in TCGED, respectively $(p<0.05)$.

Table 5. Plasma membrane function of sperm in rabbit (Mean \pm SD)

\begin{tabular}{cc}
\hline Type of extender & Tail changes $(\%)$ \\
\hline TCGD & $73 \pm 1.4^{*}$ \\
TCGED & $70 \pm 1.4$
\end{tabular}

*Different letters within same column represent a significant difference $(p<$ 0.05).

*TCGD (Tris, Citric, Glucose and DMSO), TCGED (Tris, Citric, Glucose, Ethylene glycerol and DMSO).

Table 4. Effect of 10 and $20 \%$ of egg yolk concentrations in semen extender on survival ability

\begin{tabular}{ccccccccc}
\hline \multirow{2}{*}{$\begin{array}{c}\text { Type of semen } \\
\text { extenders }\end{array}$} & $\mathbf{8}$ & $\mathbf{8}$ & \multicolumn{7}{c}{ Preservation periods $(\mathrm{h})$} \\
\cline { 2 - 8 } & $\mathbf{0}$ & $\mathbf{1 2}$ & $\mathbf{2 4}$ & $\mathbf{3 6}$ & $\mathbf{4 8}$ & $\mathbf{6 0}$ & $\mathbf{7 2}$ \\
\hline TCG10 & $86.4 \pm 0.5$ & $77.0 \pm 0.8$ & $67.5 \pm 1.9$ & $65.8 \pm 1.1$ & $48.2 \pm 1.2$ & $36.0 \pm 0.9$ & $11.1 \pm 1.7$ & 0 \\
TCG20 & $85.9 \pm 1.2$ & $76.7 \pm 1.1$ & $70.1 \pm 1.2$ & $60.8 \pm 1.2$ & $39.2 \pm 0.1$ & $19.3 \pm 1.0$ & $15.5 \pm 0.9$ & 0 \\
\hline
\end{tabular}

*TCG10 (Tris, Citric, Glucose and 10\% egg yolk), TCG20 (Tris, Citric, Glucose and 20\% egg yolk).

*Different letters within same column represent a significant difference $(p<0.05)$. 

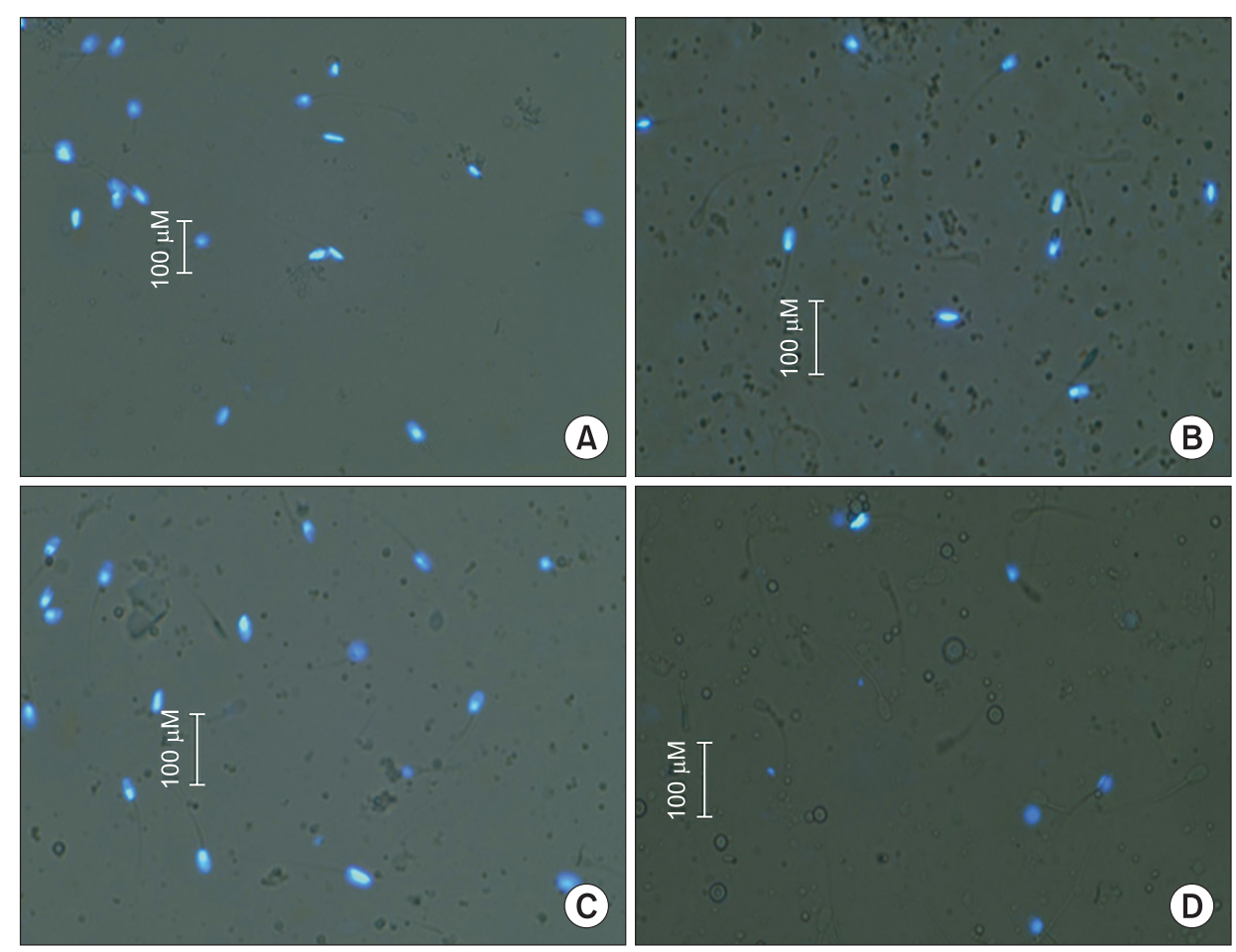

Table 6. Effect of freezing extender and thawing method on the survival ability of frozen Rabbit spermatozoa

\begin{tabular}{lllll}
\hline \multirow{2}{*}{$\begin{array}{c}\text { Type of } \\
\text { extender }\end{array}$} & Viability (\%) & \multicolumn{3}{c}{ Acrosome (\%) } \\
\cline { 3 - 5 } & & \multicolumn{1}{c}{ F } & \multicolumn{1}{c}{ B } & \multicolumn{1}{c}{ AR } \\
\hline TCGD & $46.4 \pm 2.8$ & $56.3 \pm 1.4$ & $24.8 \pm 0.3^{*}$ & $18.9 \pm 1.2^{*}$ \\
TCGED & $23.0 \pm 1.1$ & $54.6 \pm 1.4$ & $33.6 \pm 0.3^{* *}$ & $11.8 \pm 1.0$ \\
TCGGD & $53.4 \pm 0.1^{*}$ & $62.3 \pm 0.4^{*}$ & $20.3 \pm 0.7$ & $17.4 \pm 0.4^{*}$ \\
TCGSD & $61.3 \pm 0^{* *}$ & $67.1 \pm 0.1^{* *}$ & $21.1 \pm 0.7$ & $11.8 \pm 0.8$ \\
\hline
\end{tabular}

F: Normal Acrosome Intact , B: Capacitated, AR: Reactive.

*TCGD (Tris, Citric, Glucose and DMSO), TCGED (Tris, Citric, Glucose, Ethylene glycerol and DMSO), TCGGD (Tris, Citric, Glucose, Glycerol and DMSO), TCGGD (Tris, Citric, Glucose, Sucrose and DMSO).

****: Different letters within same column represent a significant difference $(p<0.05)$.

\section{DISCUSSION}

This study analyzed the effects of acrosome normality and viability of sperm to TCG (Tris, Citric acid, Glucose, Egg yolk) agent to ethylene glycerol, glycerol, sucrose, regulation of DMSO concentration is compared to the many research. According to a study by Gotzeand Paufler (1976), changes of egg yolk concentration in TCG suggested that rabbit sperm could have a great effect on frozen sperm, but the difference was not known. In the present study, $10 \%$ egg yolk supplementation resulted in a survival rate of $77 \pm 0.8$, acrosome steadiness of $73.3 \pm 0.9 \%$, and plasma membrane function of $73 \pm 14 \%$, the survival rate of long term sperm could be maintained during storage at low temperature rather than $20 \%$ egg yolk supplementation. In addition, low survival rate was recorded by cold shock in free egg yolk TCG. It was considered the survival rate would be lowered in the plasma membrane damage due to exposure to low temperatures in rabbit sperm head (Cortell and Viudes de Castro, 2008). Based on the present study, the second frozen agent by mixing the solution related to freezing preservation after the first low temperature preservation in each frozen agent using $20 \%$ egg yolk was resulted in differences in the survival rate and acrosome normality. According to Arriola (1982), glycerol on frozen of rabbit sperm wastoxic compared to other species, it could affect the survival rate (Arriola, 1982). It was recommended to use methyl, amide and DMSO as alternative materials for freeze protection but that was known to have highly no effect at living rate of sperm and changes of acrosome (Hanada and Nagase, 1980). However, the addition of DMSO and egg yolk couldhave a positive effect on the normality and survival rate of rabbit sperm. In a result study of improving IVF correction rate, the final $1.5 \%$ glycerol or $0.1 \mathrm{M}$ sucrose and $14 \%$ 
DMSO to the first frozen agent supplement 10\% egg yolk showed higher survival rates than a result of the existing $10 \%$ DMSO and $4.8 \%$ glycerol added frozen agent. It was compared to the $4.5 \%$ DMSO and $1 \%$ glycerol frozenagent of Polgar et al. (2004), high acrosome normality and survival rate were shown during sperm freezing and thawing. Compared to the methods presented in many other studies, high concentrations of DMSO and low concentrations of glycerol additions could be considered effective in protecting the plasma membrane of rabbit sperm head (Foote et al., 2000; Cortell et al., 2008). These findings are considered to have a significant effect on sperm conservation in rabbits according to the concentration control of the frozen agent.

\section{CONFLICTS OF INTEREST}

No potential conflict of interest relevant to this article was reported.

\section{ORCID}

Sang Hwan Kim: https://orcid.org/0000-0003-0996-6912 Seong-Gyu Bang: https://orcid.org/0000-0002-2513-4887 Min-Gee Oh: https://orcid.org/0000-0002-9289-3779 Chan-Hee Park: https://orcid.org/0000-0002-7229-244X Jong Taek Yoon: https://orcid.org/0000-0002-9408-8239

\section{REFERENCES}

Arriola J. 1982. Interaction if formaldehyde and of sodium and triethanolamine lauryl surfate on the motility and fertilizing ability of rabbit and bull spermatozoa frozen in the egg yolk and milk extender. Ph.D.Thesis. Cornell University. Ithaca. New York.

Arriola J, Foote RH. 1987. Glycerolation and thawing effects on bull spermatozoa frozen in detergent-treated egg yolk and whole egg extenders. J Dairy Sci. 70:1664-1670.

Chen Y, Foote RH. 1994. Survival of rabbit spermatozoa frozen and thawed at different rates with and without seeding. Anim Reprod Sci. 35:131-143.

Chen Y, Li J, Simkin ME, Yang X, Foote RH. 1989a. Fertility of fresh and frozen rabbit semen inseminated at different times is indicative of male differences in capacitation time. Biol Reprod. 41:848-853.

Chen Y, Yang X, Foote RH. 1989b. Timed breeding of rabbits with fresh and frozen-thawed semen and evidence of acrosome alteration following freezing and thawing. Anim Reprod Sci. 18:35-41.

Cortell. C, Viudes de Castro. M.P. 2008. Effect of gelatin addi- tion to freezing extender on rabbit semen parameters and reproductive performance. In: Xiccato. G. (Ed.), 9th World Rabbit Congress.327-.332.

de Leeuw AM, den Daas JH, Woelders H. 1991. The fix vital stain method. Simultaneous determination of viability and acrosomal status of bovine spermatozoa. J.Androl. MarApr;12(2):112-8.

Dewit M, Marley WS, Graham JK. 2000. Fertilizing potential of mouse spermatozoa cryopreserved in a medium containing whole eggs. Cryobiology. 40:36-45.

El-Alamy, Foote RH. 2001. Freezability of spermatozoa from Finn and Dorset rams in multiple semen extenders. Anim Reprod Sci. 65(3-4):245-254.

Foote R.H. Carney E.W. 2000. The rabbit as a model for reproductive and developmental toxicity studies. Reprod. Toxicol. 14:477-493.

Gotze VG, Paufler S. 1976. Fertility results and prenatal mortality following artificial insemination with fresh and deep frozen semen in rabbits after normal induction of ovulation and superovulation. Zuchthyg. 11:169-174.

Hammerstedt RH and Graham JK.1992. Cryopreservation of poultry sperm: The enigma of glycerol. Cryobiology. 29:2638.

Hanada A and Nagase H. 1980. Cryoprotective effects of some amides on the rabbit spermatozoa. J Reprod. Fertil. 60:247252.

Jeyendran RS, van der Ven HK, Perez-Palaez M, Crabo BG, Zaneveld LJ. 1984. Development of an assay to assess the functional integrity of the human sperm membrane and its relationship to other semen characteristics. J Reprod. Fertil. 70:219-228.

Larson JL and Miller DJ. 1999. Simple histochemical stain for acrosome on sperm from several species. Mol. Reprod. Dev. 52:445-449.

Polgar ZS, Virag GY, Baranyai B, Bodo SZ, Kovacs A, Gocza E. 2004. Evaluation of effects of cryopreservation on rabbit spermatozoa membranes with trypan blue-Giemsa staining, In: Becerril, Pro, A. (Eds.), 8th World Rabbit Congress. World Rabbit Sci. 322-329.

Polge C, Smith AU and Parkes AS. 1949. Revival of spermatozoa after vitrification and dehydration at low temperatures. Nature. 164:666.

Pursel VG, Schulman LL, Johnson LA. 1978. Effect of Orvus Es Paste on acrosome morphology, motility and fertilizing capacity of frozen-thawed boar sperm. J Anim Sci. 47:198-202.

Rota A, Stro“m B, Linde-Forsberg C, Rodriquez-Martinez H. 1997. Effects of Equex STM paste on viability of frozenthawed dog spermatozoa during in vitro incubation at $38^{\circ} \mathrm{C}$. Theriogenology. 47:1093-1101.

Sawanda Y and Chang MC. 1964. Motility and fertilizing capacity of rabbit spermatozoa after freezing in a medium containing dimethyl sulfoxide. Fertil. Steril. 15:222-229.

Watson PF. 2000. The causes of reduced fertility with cryopreserved semen, Anim. Reprod. Sci. 60-61:481-492. 